Schweiz. Z. Path. Bakt. 1959;22:I-VIII

\title{
Contents, Vol. 22, 1959
}

Offizielles Organ der FreienVereiniguiig SchweizeГ Pathologen/Schweiz. Mikrobiologischen Gesellschaft Organe officiel de ГAssociation fibre des Pathologistes Suisses/la Société Suisse de Microbiologie

H. BERGSTRAND, Stockholm

R. BIELING, Wien

H. CHIARI, Wien

M. DJURISIC, Beograd

J. FIRKET, Liège

C. HALLAUER, Bern

E. LETTERER, Tubingen

E. G. NAUCK, Hamburg

EDITORES

J. L. NICOD, Lausanne CH.OBERLING, Paris A. L. OLITZKI, Jerusalem F. C. ROULET, Basel E. RUTISHAUSER, Geneve H. SMETANA, Washington J. TOMCSIK, Basel

R. TULASNE, Strasbourg E. UEHLINGER, Zurich H. UNGAR, Jerusalem B. WALTHARD, Bern M. WELSCH, Liège A. WERTHEMANN, Basel K.C. WINKLER, Utrecht REDACTORES A. v. ALBERTINI, Zurich A. GRUMBACH, Zurich H. MOOSER, Zurich Vol. 22

1959

\section{BASEL (Schweiz) S. KARGER NEW YORK}

- Index

Agnese, G.: vide Petrilli, F. L.

Ahrens, W.: vide Fuchs, G. H. P.

Albertini, A. von: Zur Morphologie der termmalen Strombahn im Granulationsgewebe 285

Aufdermaur,M. und Brodhage, H.:

Die experimentelle Rattenlepra

363

Bächtold, H. P.: vide Zbinden, G.

Balázs, V., Tiszai, A., Kovács, K., Fröhlich, M. M. and Benkö, A.:

The Effect of Storage of Methylcellulose on the Immunehaemolysin

Formation in Rats 32

Baniõ, S.: $\quad$ Evidence of Reverse Mutation from Streptomycin Resistance to

Sensitivity in Salmonella typhimurium 511

Baumann, U.: Histologische, blutchemische und elektrophoretische Untersuchungen über die Masugmephritis der Ratte bei gleichzeitiger bakterieller In-

fektion. Zugleich ein Beitrag zur chemischen und elektrophoretischen

Normalwertbestimmung des Rattenserums 789

Baumann-Grace, J. B., Kovács, H. und Tomcsik,J.:

Extraktion von Polysaccharidhaptenen aus der Zellwand des Bacil

lus anthracis und des Bacillus cereus 158 
Baumann-Grace, J.B.: vide Tomcsik,J.

Benkö, A.: vide Balázs, V.

Berger, E.: Immunochemische Untersuchungen mittels des Haemagglutinationstestes bei Nahrungsmittel-Antigenen und Antikörpern 676

Boss, J. H.: Spontane Hypoglykämie als Folge eines nicht-insulinproduzierenden Tumors der Nebennierenregion 232

Bouille,M.: vide Tomcsik,J.

Bouvier, C. A.: $\quad$ La musculature striée des cirrhotiques

Brodhage, H.: vide Aufdermaur,M.

Bruhin, H.: vide Vischer, W. A.

Brun, R.: vide Maggiora, A.

Brunner, H. E.: vide Rüttner, J. R.

Burgherr,A.: Über einen mikrobiologischen Test auf Hepatitis epidemica . . 177

Burkholdér, P.: vide Klein, P.

Coons, A. H.: Antibodies and Antigens Labelled with Fluorescein 693

The Diagnostic Application of Fluorescent Antibodies $\quad 700$

Cottier, H., Gautier, E. und König,M. P.:

Vermehrung und Polymorphic azidophiler Zellen in der Adenohypo-

physe bei Gargoylismus 383

Crovari, P.: vide Petrilli, F. L.

Dabrowski, L.: vide Slopek, S.

Hathorn,M.: vide Gillman, Th.

Heinbich, S., Pulverek, G. und Hanf, U.:

Über das physiologische Vorkommen des Bacteroides melaninogeni-

cus bei Mensch und Tier 861

Hess, R. und Pearse, A. G. E.:

Renale Glucose-6-Phosphat-Dehydrogenase und experimenteller

Hochdruck der Ratte 329

Heumann, W.: vide Windisch, F.

Hulanicka, E.: vide Pakula, R.

Jadassohn, W.: vide Maggiora,A.

Juhász, J.: Über die Entstebung einer malignen Riesenzellengescbwulst bei

monostotischer Ostitis deformans Paget 242

Kapanci, Y. et Veyrat, R.:

Artériosclérose pulmonaire par thromboses artérielles et artériolaires 392

Kaufmann, G.: vide Wiesmann, E.

Kern, H.: vide Sackmann, W.

Kétyi,L: vide Rauss, K.

Killisch, L.: vide Poetschke, G.

Klein, P. und Burkholder, P.:

Die Darstellung von fixiertem Komplement mit markiertem Anti-

komplement 729

König,M. P.: vide Cottier, H.

Konsanszky, A.: Verwendung eines Osmometers zur Verfolgung der Veränderungen während der Fermentation 469

Konsanszky, A.: Untersuchungen über den Stoffwechsel der Hefe. I. Die Wirkung 
pflanzlicher Porphyrine auf die Vermehrung der Hefezellen ... 515

Konsanszky, A. und Fazekas,A.:

Untersuchungen über den Stoffwechsel der Hefe. II. Die Wirkung

pflanzlicher Porphyrinverbindungen auf die Ergosterinsynthese in

Hefekulturen 818

Konsanszky, A.: Untersuchungen über den Stoffwechsel der Hefe. III. Vergleichende

Untersuchungen über die Wirkung verschiedener Verbindungen auf das Wachstum und den

Ergosteringehalt von Hefekulturen .... 822

KovÁcs, E. und Pettkó, E. F.:

Vergleichende Untersuchungen der Zellatmung mit Hilfe manome-

trischer und elektrometrischer Methoden 229

KovÁcs, E.: vide Matkovics, B.

KovÁcs, H.: vide Baumann-Grace, J. B.

KovÁcs, K.: vide Balázs, V.

Kradolfer, F.: vide Gross, F.

Krech, U.: $\quad$ Der Antikörper-Nachweis bei Virusdoppelinfektionen 664

Kunz, Ch.: Weitere fluoreszenz-serologische Untersuchungen an Pilzen . 1/8, .. 742

Kubihara, M.: vide Segi, M.

Kukmann, J.: Der Selektiv-Nährboden nach Achalme zum Nachweis eiweißzer-

setzender Clostridien als Verschmutzungsanzeiger aus Wasser . . . 588

Lindner, G.: vide Petuely, F.

Löffler, H.: $\quad 1$ st das pseudoluetische (Wa. R. positive) Lungenfiltrat eine Ornithose ? 658

Maggioka, A., Bbun, R. et Jadassohn, W.:

Effet de divers bois sur certains micro-organismes in vitro .... 621

Matkovics, B., Kovács, E. und Sipos, Gy.:

Stoffwechseluntersuchungen an Streptomyces griseus - Kulturen.

II. Mitteilung: Veränderung der Sauerstofftension in Oberflächen-

und Tiefenkulturen 214

Mayersbach, H.: vide Rotter, K.

Meisel, H. und Trembowler, P.:

liber Perfringensbazillenantikörper im menschlichen Serum . . 490

Nordheim, W.: vide Windisch, F.

Olitzki, A.L., Godinger,D. and Sharon, N.:

Antigenic Relationships between Differeut Tribes of the Parvobac-

teriaceae $\quad 506$

Pakula, R., Hulanicka, E. and Walczak, W.:

Transformation Reactions Between Different Species of Streptococci and Between Streptococci,

Pneumococci and Staphylococci 202

Papacharalampous, N. X.:

Über einen seltenen Fall von «Glomustumor» des Vaguskörpers mit

Hypotonie 784

Pearse, A. G. E.: vide Hess, R.

Petrilli, F. L., Agnese, G. et Cbovabi, P.:

Utilisation des hématies hyperformolées en sérologie

Pettkó,E. F.: vide KovÁcs,E.

Petuely, F. und Lindneb, G.: 
Die Methodik der Darmfloraanalyse mit fluoreszensmarkierten Anti-körpern (Markierung mit 1Dimethylamino-Naphtalinsulfosäure-5) . 747

Poetschke, G. und Killisch, L.:

Untersuchungen mit fluoreszenz-markierten Antikörpern. VI. Fluo-reszenzserologische Untersuchungen an Spirochäten (T. pallidum, Reiter-Spirochäten), Bor. recurrens und Schleimhautspirochäten . . 765

Poetschke, G., Uehleke, H. und Killisch, L.:

Untersuchungen mit fluoreszenz-markierten Antikörpern. V. Gleichzeitiger Nachweis mehrerer Antigene durch verschieden gefärbte fluoreszierende Antikörper am Beispiel von Proteus morganii und Bacillus cereus (Hierzu Tafel III) 758

Postebnak, Th.: vide Germanier, R.

Pulveber, G.: vide Heinrich, S.

Rauss, K. and Kétyi, I.:

Determination of the Immunogenic Value of Boivin Antigens by

Means of Haemagglutination-Inhibition Test 20

Riniker, P.: Experimentelle Knochenbildung

Rondez, R.: vide Rüttner,J. R.

Rotter, K. und Mayersbach, H.:

Versuche zum Nachweis von Gewebsantikörpern bei Tuberkulose mittels immunhistologischer Methoden 732

Roulet, F. C: Über Mitosen in Leberepithelien bei Parotitis epidemica .... 112

Rüttner, J. R. und Bbunneb, H. E.:

Zur Frage der experimentellen Induktion von Tumoren des lymphatischen Systems der Ratte mit den Diazo-Farbstoffen Trypanblau

und Evans Blue 519

Rüttner, J. R.; Rondez, R. und Gassmann, R.:

Histochemische Untersuchungen an der toxisch geschädigten Ratten-

leber 294

Sackmann,W., Kebn, H. und Wiesmann, E.:

Untersuchungen über die biologischen Wirkungen von Tomatin und Solanin $\quad 557$

Scheideggeb, S.: Schwer rubrizierbare Formen der Virusencephalomyelitis 337

Schlegel-Opbecht, E.:

Untersuchungen über die Rekombination des A-Antigens nach der

Auskreuzung des bekapselten E. coli-Stammes Fe 120 mit E.coliK

$12 / \mathrm{Hfr} 593$

Schlegel-Opbecht, E. und Fey, H.:

Genetische Untersuchungen der Kapsel-Antigene bei Escherichia coli.

I. Der A-Antigen-Verlust eines Coli-Stammes und der Versuch seiner genetischen Deutung 87

Schnitzeb, A.: 1st die Prausnitz-Küstnersche Reaktion für den Nachweis von Antikörpern beweiskräftig ? 684

Scholeb, H. J.: Experimentelle Aspergillose der Maus (Aspergillus fumigatus) und ihre chemotherapeùtische Beeinflussung $\quad 564$

Schopfeb, W. H.: vide Gebmanier, R. 
Segi,M., Fujisak,u, S. and Kurihara,M*:

Mortality for Gastric and Duodenal Ulcer in Countries and its Geographical Correlation to Mortality for Gastric and Intestinal Cancer 777

Sharon, N.: vide Olitzki,A. L.

Silberberg, R. and Silbebbebg, M.:

Einfluß einer fettreichen Kost auf die Entwicklung der Arthrosis

deformans bei kastrierten Mäusen 447

Silberberg. M.: vide Silberberg, R.

Sipos, Gy.: vide Matkovics, B.

Slopek, S, and Dabrowsky, L.:

Studies on the Tercnolabile Antigens of Shigella flexneri Y . . . 12

Stanic,M.: Die physikalischen, chemischen und biologischen Eigenschaften des

Sandottergiftes 598

Sträüli, P.: $\quad$ Was bedeuten die sogenannten Vogelaugen im Krebsgewebe? . . 368

Stüder,A.: vide Zbinden, G.

Stünzi, H.: $\quad$ Zur Pathologie des Lungenkarzinoms bei Hund und Katze .... 311

Surján,M.: Large Scale Production of Tetanus Toxin, III. The Production of

Highly Potent and Pure Tetanus Toxin 224

Thölen,H.: vide Gloor, F.

Tirunarayanan, M. 0.: vide Vischer, W. A.

Tiszai, A.: vide Balázs, $\mathrm{V}$.

Tomcsik, J. und Baumann-Grace, J. B.:

Serologische Typen von Bacillus cereus und ihre Verwandtschaft

mit Bacillus anthracis 144

Tomcsik, J., Bouille,M. et Baumann-Grace, J.B.:

Reaction spécifique de Гexosporium chez Bacillus Cereus et Bacillus

Anthracis 630

Tomcsik, J.: vide Baumann-Grace, J.B.

Trembowler, P.: vide Meisel, $\mathrm{H}$.

Uehleke, H.: Untersuchungen mit fluoreszenz-markierten Antikörpern. IV. Die

Markierung von Antikörpern mit Sulfochloriden fluoreszierender

Fárbstoffe $\quad 724$

Uehleke, H.: vide Poetschke, G.

Vajda, Gy. : Phagocytosis of Trichomonas Vaginalis $\quad 858$

Veyrat, R.: vide Kapanci, Y.

Vischer, D.: Serologische Untersuchungen an Staphylokokken 42

Vischer, W. A., Tirunarayanan, M. 0. und Bruhin, H.:

Neue chemische Methode zur raschen Unterscheidung Isoniazid-sen-

sibler und Isoniazid-resistenter Tuberkelbakterien $\quad 577$

Walczak,W.: vide Pakula, R.

Weinbach, R.: Die Verwe $\pi$ dbarkeit formolbehandelter Erythrocyten als Antigen-

träger in der indirekten Haemagglutination. II. Mitteilung ...1

Werthemann, A.: Über Spätschäden verschiedener Organe durch Thorotrast und autoradiographischer Nachweis desselben 350

Weyer, $\mathrm{F} \cdot$ : Experimente mit einem Stamm von Zeckenbißfieber aus Nordqueensland 609 
Wiesmann, E. und Kaufmann, G.:

Serologische Befunde bei Adenovirus-Infektionen, Ornithose und Lues

(2 Mitteilung.) 653

Wiesmann,E.: vide Sackmann, W.

Windisch, F., Nordheim,W. und Heumann,W.:

Anoxygene Reaktivierung des anabiotisch sistierten Phosphatwech-sels zwischen Medium und Zytoplasma. II. Mitteilung : Wirkung von Dekokten aus tierischen und menschlichen KreL·sgeweben . . 458

Wöckel, W•: Polyradiculitis nach Poliomyelitisvaccination 807

Wolf, L.: Elektronenmikroskopische Untersuchung an Ehrlich-Ascites-Tumor-

zellen. Nachweis von virusartigen Körpern in den Tumorzellen . 475

Zangger, J.: Über die Verwendung von «Aetidion» in der medizinisch-bakterio-

logiscben Diagnostik 536

Zbinden, G., Bächtold, H. P. und Studer. A.:

Zur Histopathologie experimenteller unspezifischer Entzündungen

der Rattenpfote 300

Zollinger, H. U.: Die hypertensive Arteriolopatbie 262

Gesellschaftsberichte - Society Transactions - Sociétés

Association Libre des Pathologistes Suisses. 24eAssemblée annuelle

à Neuchâtel, les 21 à 23 novembre 1958. - Freie Vereinigung der

Scbweizer Pathologen. 24. Jahresversammlung in Neuenburg, 21.

bis 23. November $1958 \quad 261$

Schweizerische Mikrobiologische Gesellschaft. 18. Jahresversammlung, 27./28. Juni 1959, in St.

Gallen. - Société Suisse de Microbio-logie. 18e assemblée annuelle, les 27 et 28 juin 1959, à St-

Gall . . 545

MiTGLIEDERLISTE DER SCHWEIZERISCHEN MiKROBIOLOGISCHEN

GeSELLSCHAFT. - MeMBRES

DE LA SOCIÉTÉ SüISSE DE MICROBIOLOGIE 771

Nachruf - Necrologue - Obituary

Dr. Dr. h. c. Heinz Karger 257

Buchbesprechungen - Book Reviews - Livres Nouveaux . . . 123, 252, 398, 539, 870

Register rerum 875

Register autorum $\quad 894$

Alle Rechte, insbesondere das der Übersetzung in fremde Sprachen, vorbehalten. Ohne ausdГückliche Genehmigung des Verlages ist es auch nicht gestattet, dieses Buch oder Teile daraus auf photomechanischem Wege (Photokopie, Mikrokopie) zu •vervielïältigen.

(C)

Copyright 1959 by S. Karger A.G., Basel Printed in Switzerland by Buchdruckerei Friedrich Reinhardt AG.( Basel Cliches: Aberegg-Steiner \& Cie. AG., Bern, und Steiner \& Cie. AG., Basel 